\title{
Improving funding and publishing workflows with PIDs
}

\author{
Gabriela Mejias* \\ ORCID Engagement Manager, Europe, Middle East and Africa \\ ORCID: http://orcid.org/0000-0002-1598-7181
}

\begin{abstract}
Tracking and understanding the impact of research is important to funders, publishers, and researchers. But the process of doing so is full of pain points - from the time it takes to apply for a grant and report on it, to the often poor data quality of the information being collected, and the challenge of keeping track of researchers and their research over time. The ORCID Funder Working Group recently analyzed these pain points and published their recommendations for using persistent identifiers to help address these challenges. In this article, you will learn more about persistent identifiers for researchers, publications, and grants and about what each stakeholder group can do to reduce the burden and improve the transparency of the process - from applying for a grant to publishing the research results.
\end{abstract}

Keywords: Persitent identifiers, research infrastructure, scholarly communication, funders, publishers, interoperability

\section{ORCID and interoperability}

ORCID's vision is a world where all those who participate in research, scholarship, and innovation are uniquely identified and connected to their contributions and activities across organizations, disciplines, borders and time [1].

ORCID provides open tools (ORCID iDs, Registry and APIs) that can be integrated into systems to make transparent trustworthy connections between people, organizations, contributions and activities that everyone can benefit from. We rely on our community and members to achieve more interoperability (Fig. 1) and build a robust and open research infrastructure. Researchers are at the center of our interoperability vision connecting their iD with publishers, funders, and research institutions. organizations play their part by integrating ORCID APIs to enable automatic exchange of research data between systems and sharing information about the connections between them and their researchers. For research institutions, this means validating their researchers' affiliation(s); for publishers, their works (publications, reviews, datasets, and more); and for funders, their grants and awards.

Persistent identifiers (PIDs) are a key element of this community approach. Together with ORCID iDs for individuals and DOIs for research outputs, there are other useful PIDs to make research data FAIR [2] and address some of the challenges in funding and publishing workflows:

*E-mail: g.mejias@orcid.org. 


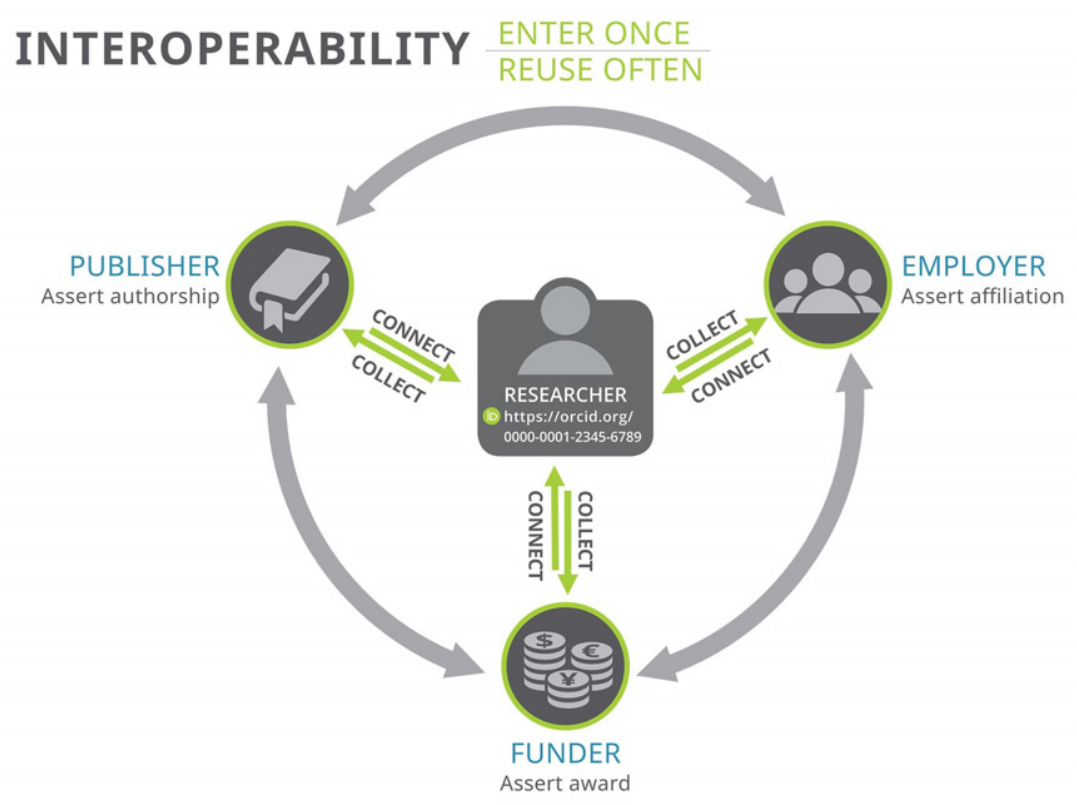

Fig. 1. ORCID interoperability vision. Published under a CC0 license.

- Funder IDs: for organizations that fund research. The Open Funder Registry is managed by Crossref as a unique taxonomy of grant-giving organizations for published scholarly research. The Registry was launched in 2012 with data donated by Elsevier. The Registry is available under a CC0 license and data search and REST API are free to use [3].

- ROR IDs: for research organizations. The Research Organization Registry (ROR) is a communitydriven initiative to develop an open, intererable, unique identifier for every research organization in the world. The initial steering group consisted of California Digital Library, Crossref, DataCite, and Digital Science and tasked with implementing the pilot project, ingesting donated data from GRID to the registry. ROR was officially launched in 2019. All ROR IDs and metadata are available under a CC0 license. ROR search, API, reconciler and data dump are freely available [4].

- Grant IDs: unique identifiers (DOIs) to register grants, and allow researchers to include this information in their submissions to publishers and data repositories. Grant IDs are managed by Crossref and the result of their Funder advisory group discussions between 2017 and 2019 [5]. The first grant IDs were issued in 2019.

\section{ORCID engaging with funders: Working group, survey and recommendations}

The ORBIT project was launched in late 2017 to help us achieve our vision: to identify and connect researchers with their research activities. The working group gathered 21 organizations from all over the world and enabled us to engage directly with the funding community about the use of ORCID and other persistent identifiers in grant management workflows [6]. Funders have long been supportive of ORCID goals, and are now starting to adopt ORCID into their systems. 
As part of the working group, we've conveyed a survey. Thirteen funders from nine countries in six continents participated and the survey report [7] indicates the following findings:

(1) Reporting is an exercise in connecting researchers, funding, research activities and contributions, to understand the impact of investment and to monitor compliance with Open Access or research datasharing policies. Survey respondents stated that their major challenges are connecting these points, whether due to poor quality data or lack of data.

(2) Most respondents indicated that the majority of their reporting requests are fulfilled but often delivered late and substantial staff time to clean up required.

(3) Consistently linking grants and research outputs is outlined by most respondents as the best method of improving reporting.

(4) More than $50 \%$ of surveyed funders interact with researchers during the reporting process, and $60 \%$ have already integrated or in the process to integrate ORCID into their systems. This creates a great opportunity for ORCID as a tool to enhance reporting workflows.

Based on the results of the survey, the working group elaborated the following recommendations [8]:

- Funders, publishers, and identifier providers should work together to develop, and promote workflows that integrate identifiers to create and share transparent connections between individuals, funding, and research activities in grant and publication processes.

- Funder reporting systems should implement workflows to reduce reporting burden, by allowing researchers to populate web forms with data from other systems, including ORCID records, avoiding manual data entry.

- Funders should cooperate with publishers to leverage identifiers for people, organizations, grants, to facilitate compliance with funder open access and data-sharing requirements.

\section{PIDs in funding and publishing workflows}

These recommendations can be translated into the following suggested workflows to integrate PIDs in funding and publishing process to improve reporting and consistently link grants and research outputs in a transparent way:

Funding workflows

(1) Researcher registers an ORCID iD

(2) Research organization asserts employment affiliation data with a ROR ID to the researchers' ORCID record.

(3) When applying for a grant, the researcher is asked to connect the ORCID iD to the funding application system. After the iD is connected, the funder imports career and CV data from the researchers' ORCID record.

(4) Once the funding decision has been made, the funder registers a grant ID and asserts the award data to the researchers' ORCID record.

This type of implementation can enable transparent and authoritative connections between funders and researchers and save both funders' administrative staff and researchers a lot of time by avoiding manual entry or editing of information. 


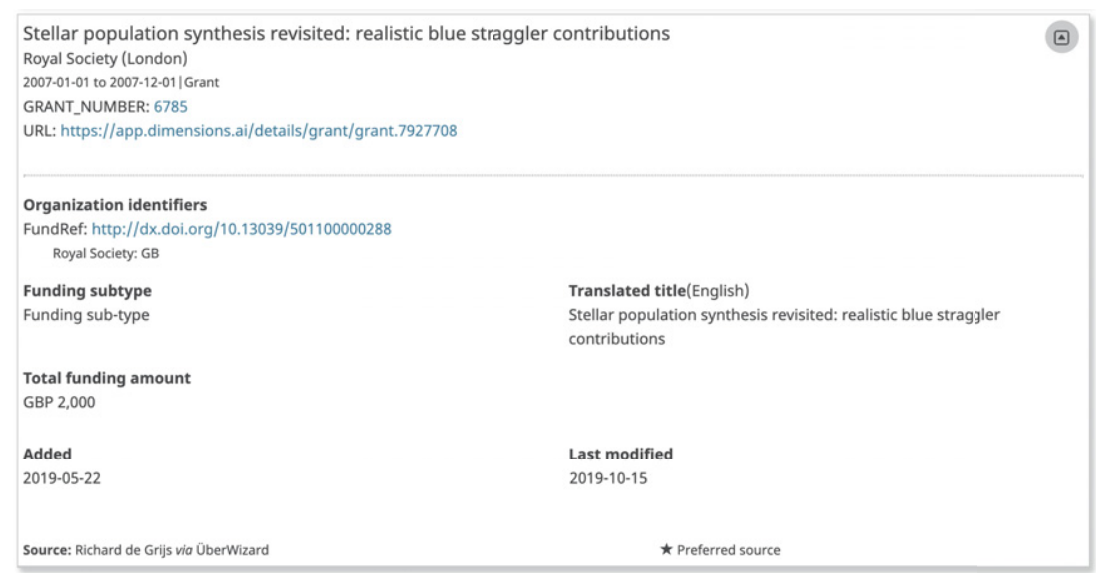

Fig. 2. Example of a funding item added to the ORCID iD https://orcid.org/0000-0002-7203-5996.

\section{Publishing workflows}

(1) When submitting a manuscript, the author is asked to connect the ORCID iD with the submission system.

(2) Publisher obtains the iD and reads the ORCID record for funding and employment data.

(3) The Publisher creates automatic funding acknowledgement (adds the grant ID) to the article metadata when registering a DOI for a publication.

(4) Publisher checks if an Open Access policy applies and publishes in line with it.

(5) Publisher connects article metadata (and links the DOI) to the authors' record.

The benefits of this approach are to correctly identify authors while creating and connecting funding acknowledgement in the article metadata. In addition, this workflow can support funders policy compliance and increase transparency by including identifiers in publication metadata.

To date 35 funding agencies have joined ORCID as institutional members [9], with more than 25 member systems integrating ORCID iDs and and exchanging data with our Registry. Individuals can automatically import grants and awards data from Überwizard (Dimensions, former Überresearch) into their ORCID records (Fig. 2). CC Grant Tracker (CC Technology) integrates ORCID and allows researchers to complete application forms by importing the data from their ORCID records. When a grant is awarded, CC Grant Tracker automatically populates the lead applicant's ORCID record with details of the grant [10]. Currently 12 funders have signed our Funders Open Letter committing to implement ORCID in their workflows in accordance with our best practices [11].

Building a robust and open research infrastructure is a community effort where funders, publishers, identifiers providers and researchers play an important role. Implementing PIDs in funding and publishing workflows can help generate better and linked research data, improve reports quality, save time by automatizing data exchange, and overall promote interoperability across systems and organizations.

\section{References}

[1] Our vision, ORCID, https://orcid.org/node/8, accessed March 152020.

[2] The Fair Data Principles, Force11, https://www.force11.org/group/fairgroup/fairprinciples, access March 152020. 
[3] The Open Funder Registry, Crossref, https://support.crossref.org/hc/en-us/articles/214360886-The-Open-FunderRegistry, accessed March 152020.

[4] Alice Meadows, Are you ready to ROR? An inside look at this new organization identifier registry, The Scholarly Kitchen (2019), https://scholarlykitchen.sspnet.org/2019/12/04/are-you-ready-to-ror-an-inside-look-at-this-new-organizationidentifier-registry/, accessed March 152020.

[5] Research grants, Crossref, https://www.crossref.org/services/content-registration/grants/, accessed March 152020.

[6] The ORBIT project, ORCID, https://orcid.org/organizations/funders/orbit, accessed March 152020.

[7] Alice Meadows, Josh Brown, Tom Demeranville and Haak Laurel, ORBIT Funder Reporting Survey Report, figshare, 2019, Online resource, doi:10.23640/07243.9149240.v1.

[8] ORCID Funder Working Group, ORCID and Grant DOIs: Engaging the Community to Ensure Openness and Transparency of Funding Information, figshare, 2019, Online resource, doi:10.23640/07243.9105101.v1.

[9] ORCID Member organizations, ORCID, https://orcid.org/members, accessed March 152020.

[10] Tom Demeranville, BLOG: ORCID and Funder Workflows - An Update, figshare, 2019, Online resource, doi:10.23640/ 07243.8397554.v1.

[11] ORCID Funders Open Letter, ORCID, https://orcid.org/organizations/funders/open-letter, accessed March 152020. 